\title{
Elemental Mapping of NiTi with EFTEM
}

\author{
J.E. Wittig, ${ }^{*}$ J. Bentley, ${ }^{* *}$ N.D. Evans, ${ }^{* *}$ Ch. Somsen, ${ }^{* * *}$ and G. Eggeler*** \\ * Department of Electrical Engineering, Vanderbilt University, Nashville, TN 37232 \\ ** Metals \& Ceramics Div., Oak Ridge National Laboratory, PO Box 2008, Oak Ridge, TN 37831 \\ *** Institut für Werkstoffe, Ruhr University, Bochum, Germany
}

Martensitic transformations in Ni-rich NiTi shape memory alloys take place as multistage transformations [1]. In Ni-rich alloys with an austenitic B2 matrix, coherent $\mathrm{Ni}_{4} \mathrm{Ti}_{3}$ precipitates form from thermo-mechanical processing and affect the sequence of the martensitic transformation. Any composition inhomogenieties that develop during the evolution of the $\mathrm{Ni}_{4} \mathrm{Ti}_{3}$ precipitates will have a large influence on the multistage martensitic transformations, since the martensite start temperature, $\mathrm{M}_{\mathrm{s}}$, is strongly dependent on the Ni concentration of the matrix [2]. Since concentration differences on the order of 0.5 at $\%$ are sufficient to influence the transformation, providing sufficiently accurate concentration profiles for meaningful structure-property correlations is a challenging experiment [3]. This investigation employs elemental mapping by energy-filtered transmission electron microscopy (EFTEM) to attempt to measure the concentration profiles at these precipitate-matrix interfaces.

A single crystal sample of $\mathrm{Ni}_{51} \mathrm{Ti}_{49}$ was annealed for $4 \mathrm{ks}$ at $550^{\circ} \mathrm{C}$ in compression along $<111>$ to selectively grow a single $\mathrm{Ni}_{4} \mathrm{Ti}_{3}$ variant [4]. The sample was sliced normal to [110] so that the precipitates are approximately on edge. Samples for EFTEM were originally electropolished to electron transparency. Unfortunately, the EFTEM results were compromised by surface films and the presence of large thickness variations from selective chemical attack. Low-angle ion milling improved specimen quality. The EFTEM elemental mapping used a Philips $\mathrm{CM} 30$ ( $\mathrm{LaB}_{6}$ cathode) equipped with a Gatan imaging filter ( $30-\mathrm{eV}$ slits, collection half-angle $\beta=4.8 \mathrm{mrad}, 0.6 \mathrm{~nm}$ pixels in $512^{2}$ images $(2 \mathrm{x}$ binning)). For Ti mapping, a standard three window method used 15-s exposures for the component images. To compensate for the lower signal of the $\mathrm{Ni} \mathrm{L}_{23}$ edge, a DigitalMicrograph custom script for segmented acquisition summed five 15-s exposures for each component image.

Figure 1 is a background-subtracted $\mathrm{Ti}$ map where a 15-s exposure of the component images produced 3,000 to 4,000 counts/pixel. In figure 2, segmented acquisition with a total acquisition time of $75 \mathrm{~s}$ for each component image resulted in pixel intensities of 4,000 to 5,000 counts in the background-subtracted $\mathrm{Ni}$ map. Figure 3 shows that $\mathrm{t} / \lambda \sim 0.25$ for these data $(\mathrm{t}$ is specimen thickness and $\lambda$ is the inelastic scattering mean-free-path, $\sim 140 \mathrm{~nm}$ for NiTi). The profiles inserted into the figures from a $30 \mathrm{~nm}$ wide $\mathrm{Ni}_{4} \mathrm{Ti}_{3}$ precipitate in the center of the images are taken from the indicated rectangular regions [60 $\mathrm{nm}$ long and averaged over 50 pixels $(30 \mathrm{~nm})$ ] where left-to-right in the profile corresponds to top-to-bottom in the image. Dividing the Ti-map by the Ni-map, to first order, removes any diffraction contrast, local thickness and incident intensity variations, and yields the ratio image shown in figure 4 . In figures 1 and 4 there is an indication of slight Ti enrichment at the interface. Although we selected our EFTEM conditions with care, additional work seeking more definitive evidence for possible concentration gradients will incorporate modeling and simulations, both to truly optimize experimental conditions and also to explore detectability levels [5].

[1] J. Khalil Allafi, X. Ren, G. Eggeler, Acta Mater. 50 (2002) 793-803

[2] J. Khalil-Allafi, A. Dlouhy, G. Eggeler, Acta Mater. 50 (2002) 4255-4274

[3] M.C. Carroll, Ch. Somsen, G. Eggeler, Scripta Mater. 50 (2004) 187-192

[4] D.Y. Li and L.Q. Chen, Acta Mater. 45 (1997) 471-479

[5] Research at the ORNL SHaRE User Facility (JB, NDE) supported by the Division of Materials Sciences and Engineering, Office of Basic Energy Sciences, U.S. Department of Energy, under contract DE-AC05-00OR22725 with UT-Battelle, LLC. 


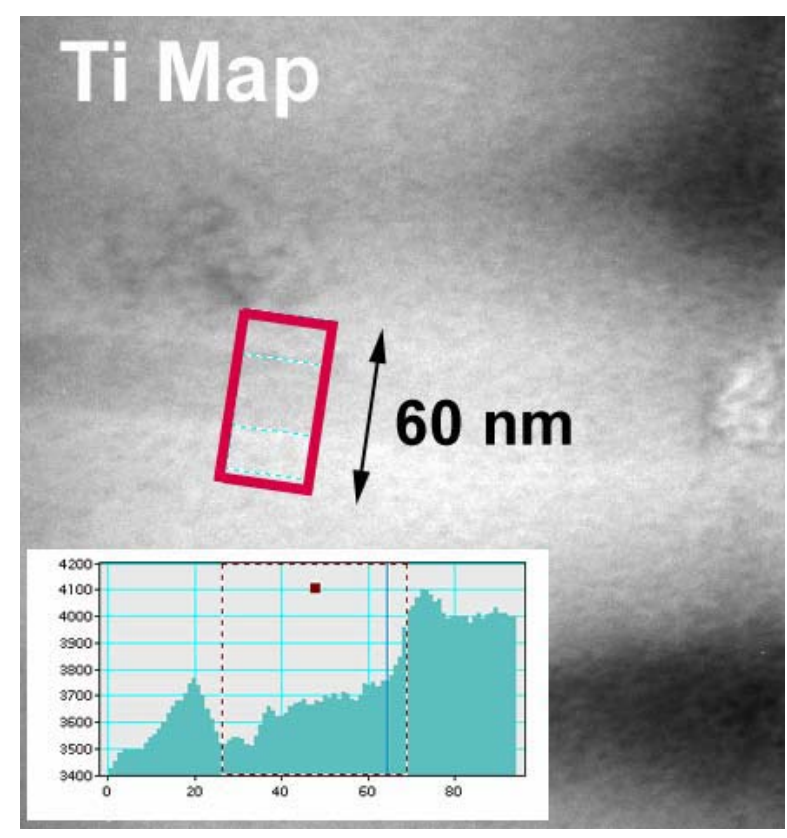

FIG. 1. The Ti map and intensity profile show a $\mathrm{Ni}_{4} \mathrm{Ti}_{3}$ precipitate $\sim 30 \mathrm{~nm}$ thick in the center of the image (left-to-right in the profile corresponds to top-to-bottom in the image).

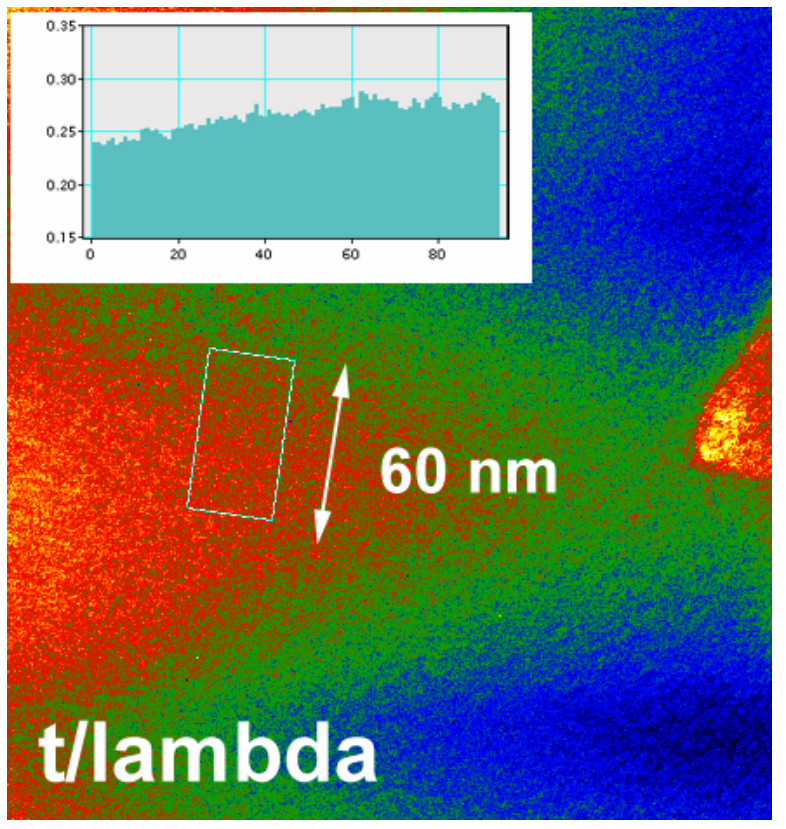

FIG. 3. The $t / \lambda$ map $(\lambda$ - the inelastic scattering mean-free-path $\sim 140 \mathrm{~nm}$ for $\mathrm{NiTi}$ ) indicates that the sample thickness ( $t$ ) is $\sim 35 \mathrm{~nm}$ for the composition profiles.

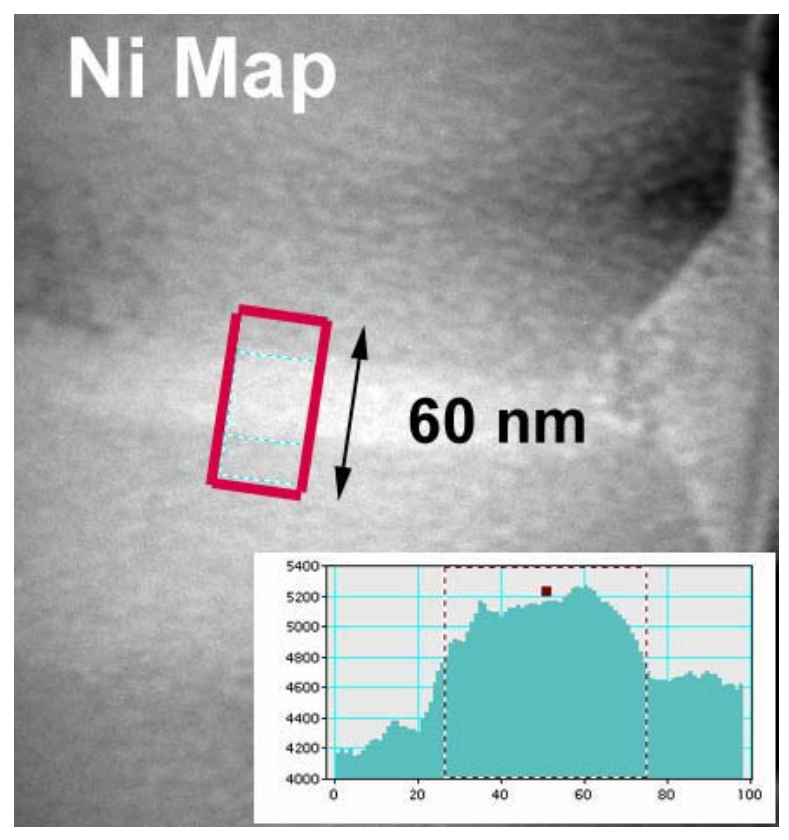

FIG. 2. The Ni Map reveals Ni enrichment in the $\mathrm{Ni}_{4} \mathrm{Ti}_{3}$ precipitate. Total acquisition time of 75-s for the component images resulted in pixel intensities of over 5,000 counts above background.

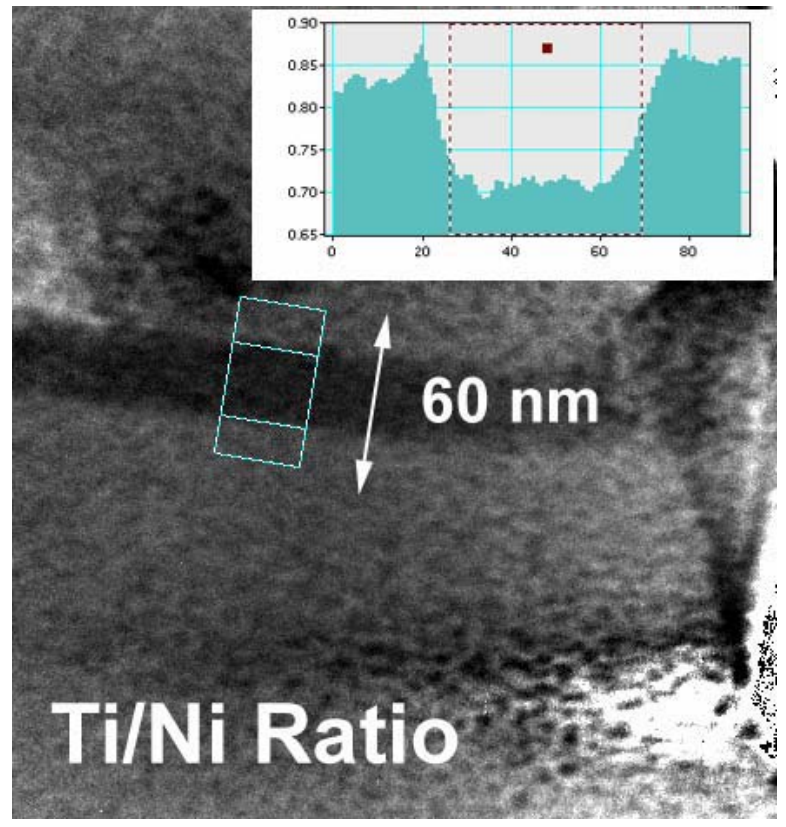

FIG. 4. Dividing the Ti map by the Ni map, which to first order removes diffraction contrast, local thickness and incident intensity variations, suggests slight $\mathrm{Ti}$ enrichment at the precipitate/matrix interface. 